\title{
Security and Privacy of Patient Information in Medical Systems Based on Blockchain Technology
}

\author{
Wu, Hongjiao; Dwivedi, Ashutosh Dhar; Srivastava, Gautam
}

\section{Published in:}

Acm Transactions on Multimedia Computing, Communications, and Applications (tomm)

Link to article, DOI:

$10.1145 / 3408321$

Publication date:

2021

Document Version

Peer reviewed version

Link back to DTU Orbit

Citation (APA):

Wu, H., Dwivedi, A. D., \& Srivastava, G. (2021). Security and Privacy of Patient Information in Medical Systems Based on Blockchain Technology. Acm Transactions on Multimedia Computing, Communications, and Applications (tomm), 17(2s), 1-17. [60]. https://doi.org/10.1145/3408321

\section{General rights}

Copyright and moral rights for the publications made accessible in the public portal are retained by the authors and/or other copyright owners and it is a condition of accessing publications that users recognise and abide by the legal requirements associated with these rights.

- Users may download and print one copy of any publication from the public portal for the purpose of private study or research.

- You may not further distribute the material or use it for any profit-making activity or commercial gain

- You may freely distribute the URL identifying the publication in the public portal 


\title{
Security and Privacy of Patient Information in Medical Systems based on Blockchain Technology
}

\author{
HONGJIAO WU, Henan Vocational College of Turna, China \\ ASHUTOSH DHAR DWIVEDI, DTU Compute, Technical University of Denmark, Denmark \\ GAUTAM SRIVASTAVA*, Brandon University, Canada, Asia University, Taiwan, and China Medical \\ University, Taiwan
}

\begin{abstract}
The essence of "blockchain" is a shared database in which information stored is un-falsifiable, traceable, open and transparent. Therefore, in order to improve the security of private information in medical systems, this paper uses blockchain technology to design a method to protect private information in medical systems and effectively realize anti-theft control of private information. First, the POPPAC model is introduced into the access control process of private information in medical systems. Next, a private information storage platform is built by using blockchain technology, and information transmission is realized using standard cryptographic algorithms. In this process, file authorization contracts are also used to guarantee the security of private information and further prevent theft of medical private information. Our simulation results show that the storage response time of this method is kept below 1000 milliseconds, and the maximum information throughput rate reaches $550 \mathrm{kbit} / \mathrm{sec}$, which indicates that this method has strong performance in information storage and transmission efficiency. Moreover, the reliability and bandwidth utilization of data transmission across domains is higher, so the method has higher information security control performance and superior overall performance.
\end{abstract}

CCS Concepts: • Security and privacy $\rightarrow$ Distributed systems security; File system security; • Information systems $\rightarrow$ Data management systems; • Networks $\rightarrow$ Network design principles;

Additional Key Words and Phrases: Blockchain; Medical System; Privacy Information; Anti-theft; Cloud Database; Asymmetric Encryption

\section{INTRODUCTION}

In current medical institutions, the degree of intelligent medical systems is becoming more common, and private information in medical data faces two major risks: information disclosure and information tampering [24, 28]. Especially in the patient's electronic medical record (EMR), there is a great deal of private information, such as patient's basic information, examination symptoms, medical order information, non-text examination information (including ultrasound images, MRI images, etc.) and other pertinent medical records [27]. All of this private information will be stored on the server of the hospital or within a hospital network which may include a network of hospitals run by the same parent organization. All data that is stored is valuable both intrinsically and medically as well. They not only provide a basis for current doctors to make judgements on the condition of patients, but also provide basis and reference for other medical practitioners to make correct assessments on the condition of patients, understand historical treatment plans, and further develop personalized treatment plans [31]. A reliable, safe and accessible medical system will

${ }^{*}$ CORRESPONDENCE: srivastavag@brandonu.ca

Authors' addresses: Hongjiao Wu, whjrpf@163.com, Henan Vocational College of Turna, Luoyang, China; Ashutosh Dhar Dwivedi, adhdw@dtu.dk, DTU Compute, Technical University of Denmark, Lyngby, Denmark; Gautam Srivastava*, srivastavag@brandonu.ca, Department of Mathematics and Computer Science, Brandon University, Brandon, Canada, College of Information and Electrical Engineering, Asia University, Taichung, Taiwan, Research Centre for Interneural Computing, China Medical University, Taichung, Taiwan. 
undoubtedly improve the search speed of medical documents which is undoubtedly beneficial to develop accurate and fast methods for patient treatment $[19,33]$.

With the development of Internet technologies such as cloud computing and cloud storage, more and more medical institutions store their medical data on cloud storage, using electronic medical records (EMR), which are most commonly hosted by third-party cloud services [3]. This approach to storage effectively solves the problem of sharing EMR among different institutions and regions. But at the same time, cloud EMR storage also has an increased chance of medical private information (MPI) leakage if MPI is stolen, hacked, tampered with. Therefore, it is essential to implement effective protection of MPI in any modern medical system.

\subsection{Related Work}

Presently, there are some relatively mature privacy information protection methods. Malliaros et al. designed a security and privacy enhancement technology to complete user authentication through open ID connect and user authentication protocols, as well as provide a reliable data access mechanism for medical information security [20]. Alghazo et al. proposed a method to protect patients' privacy data [1]. The authors presented a method that uses an encrypted watermark in patients' medical data to complete the fast and secure encryption of patients' information and protect the security of patients' privacy data. Additionally, there are protection methods for private information based on a security game model, privacy information protection methods based on spatial outlier detection, and privacy information protection methods based on information entropy suppression. However, the above presented methods have been shown to be ineffective in real-world settings where protocols need to show efficacy for large amounts of data being shared in Internet of Things (IoT) environments in real-time.

Blockchain technology is a decentralized distributed storage technology, which is characterized by non-tampering, de-trust and anonymity, and can realize data sharing, coordination, and cooperation of decentralized credit in the network [4, 11, 12, 21]. The security and privacy of blockchain technology can effectively protect the privacy information and prevent it from being stolen [ 5 , $8,16,26,30]$. Therefore, this paper present a blockchain technology-based control method for preventing theft of MPI in medical systems, which can effectively guarantee the security of private information of patients on the basis of improving the transmission efficiency of private information in the medical system.

\section{METHODS TO DESIGN}

\subsection{POPPAC model}

Patient Oriented Privacy Preserving Access Control (POPPAC) model is a model that can handle privacy protection and access control of MPI. It can better solve the problem of MPI disclosure and abuse, and meet the personalized privacy protection needs of different patients.

The POPPAC model not only solves the problem of "who operates what?" information, but also solves the problem of "what users have access to information?". Therefore, this paper introduces the POPPAC model $[13,32]$ for use in medical systems. The medical system, also known as a Health Information System (HIS), needs to deal with a large number of patients' data. Moreover, doctors, medical staff and patients need to access/view any given patients MPI, therefore the access control of patients' MPI is particularly important. In order to prevent MPI leakage, in the access control of MPI we introduce the POPPAC model that can easily realize reasonable judgement of different user roles and rights granting of access authority $[18,22]$. The specific application system architecture of the POPPAC model is shown Figure 1. 




Fig. 1. Application architecture of POPPAC model

The flow as Figure 1 follows: save the patient's cloud information in the storage area, which can be accessed by the system administrator. Relevant doctor information and cases are also input into the model. The patient cloud information is stored in the privacy information storage module of medical system based on blockchain and cloud data. In addition to the patient cloud information, the information of doctors, medical staff and doctors' laboratories are also input into the model. In the final model, the information includes the basic information of patients, medical diagnosis information, medical report information and medical laboratory data. According to the input information, the model analyzes the expected purpose of the input privacy information through the personal privacy policy management module and the general security policy management, after the inquiry and the access purpose analysis, completes the reasonable judgement of different user roles, and feeds back the judgement results to the patient privacy information access authorization module through the personalized authorization rules and the general Authorization rules grant correct access to different user roles.

\subsection{Medical system privacy information storage platform}

Due to the limited storage capacity of a general medical system, it is impossible to store all MPI locally within a hospital. Therefore, in the design of this platform, only the metadata and summary of MPI, the session key of the information provider, the signature of the information provider, and reference to the medical record in the cloud database are saved locally. All other EMR information is saved on the cloud database and hosted by a third-party cloud service organization [14, 17, 25] When data stored on cloud, hash value of that data is generated and this hash value is also stored on blockchain. In such case if an adversary modify the data on cloud it can be easily traceable. The blockchain is also used to store all events of data in the form of transaction and therefore whoever play with the data or change the data, the information is stored on blockchain and can be traceable. The architecture of this medical system MPI storage platform based on cloud storage and blockchain is shown Figure 2. 


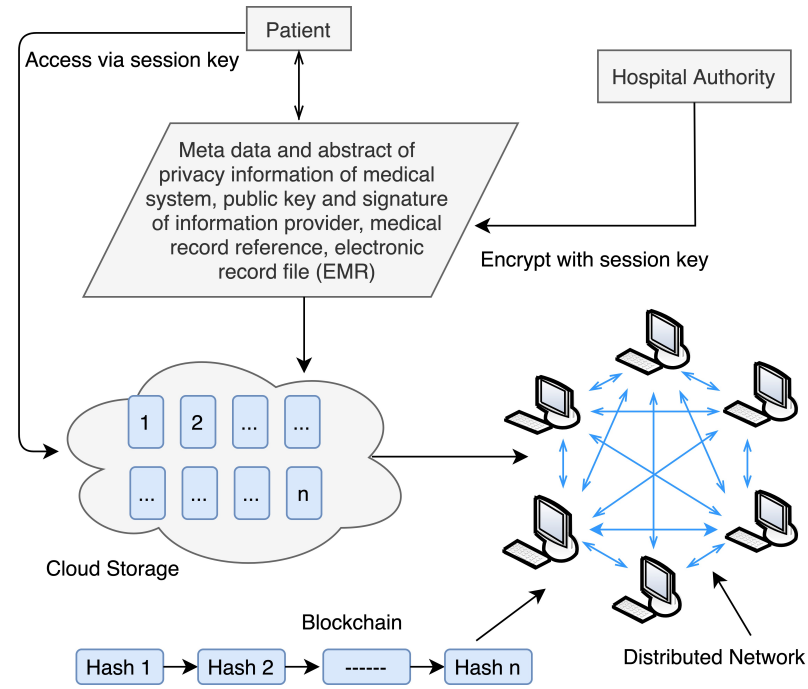

Fig. 2. Architecture diagram of privacy information storage platform of medical system

After applying cloud computing technology to the MPI platform of medical systems, the medical health platform can ensure the safe and efficient storage of patients' MPI [3, 29]. Based on session key encryption, shared access is an important design method of any MPI platform in medical systems. Hospitals and authoritative institutions generate the session key with patients and use this key to encrypt MPI $[2,15]$. Patients can access MPI through the same session key. At the same time, because the cloud platform has the characteristics of high security, privacy protection, high redundant storage, and centralization, it can prevent the loss of MPI caused by attacks or improper rights management, making MPI more secure and reliable [13]. The hash and event data is stored on blockchain and therefore any changes in the data can be detectable. Figure 3 defines the logical flow of the system.

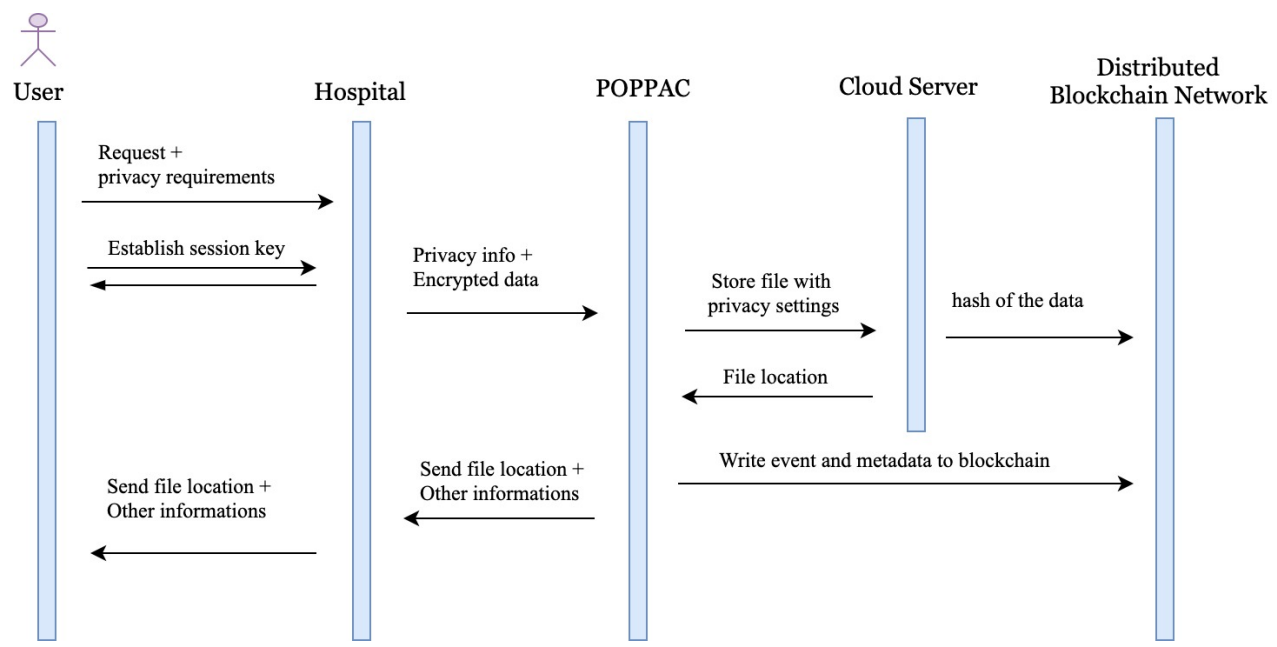

Fig. 3. Logical flow execution of the system 


\subsection{Cryptographic Set-Up}

In the proposed model, symmetric algorithms are used for the encryption of EHR. A symmetric algorithm (Private key encryption) uses a same key (see Figure 4) for encryption and decryption while asymmetric encryption (Public key encryption) uses key pair.

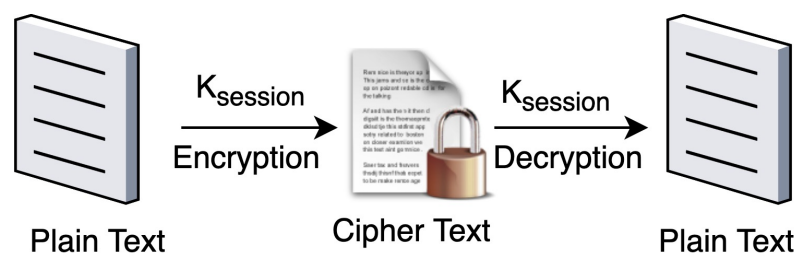

Fig. 4. Symmetric Key Encryption

The most standard and secured algorithm used for the encryption in the proposed model is the Advanced Encryption Standard (AES). AES [6] was designed by two Belgian cryptographers, Vincent Rijmen and Joan Daemen. The algorithm was the winner of the competition by the US National Institute of Standards and Technology (NIST). AES algorithm has a 128-bit block size with three supported key lengths: 128, 192 and 256 bit. Each round in AES consists of 4 layers, namely: key addition, byte shift, shift row and mix column. However, last round has only three-layers, namely: key addition, byte shift, shift row. The reason to use AES in the model is because it provides the highest security level with the smallest key size, as shown in Table 1. AES uses 80-bit key to provide the security level of 80 bit, while other algorithms use a key size of 160-bit and 1024 bit to provide the same security level. This means if a computer wants to break the 80-bit key system using exhaustive search, then it requires $2^{80}$ computational operations to do so, which becomes infeasible for malicious entities.

Table 1. Security level of cryptosystem with several key length

\begin{tabular}{|l|l|l|l|l|l|}
\hline Algorithm Family & Cryptosystem & \multicolumn{4}{|c|}{ Security Level with (k bit) key } \\
& & 80 & 128 & 192 & 256 \\
\hline Symmetric & 3DES, AES & $80 \mathrm{bit}$ & $128 \mathrm{bit}$ & $384 \mathrm{bit}$ & $512 \mathrm{bit}$ \\
\hline Elliptical Curve & ECDSA, ECDH & $160 \mathrm{bit}$ & $256 \mathrm{bit}$ & $384 \mathrm{bit}$ & $512 \mathrm{bit}$ \\
\hline Discrete logarithm & Elgamal, DSA, DH & $1024 \mathrm{bit}$ & $3072 \mathrm{bit}$ & $7680 \mathrm{bit}$ & $\begin{array}{l}15360 \\
\text { bit }\end{array}$ \\
\hline Integer factorization & RSA & $1024 \mathrm{bit}$ & $3072 \mathrm{bit}$ & $7680 \mathrm{bit}$ & $\begin{array}{l}15360 \\
\text { bit }\end{array}$ \\
\hline
\end{tabular}

In symmetric key encryption, the same session key is used to encrypt and decrypt the data and therefore choosing an efficient and lightweight key exchange algorithm is another major challenge. There are several key exchange algorithms available in public key cryptography e.g. Elgamal and Diffie-Hellman are based on discrete logarithms and RSA that is based on integer factorization. However, the most efficient algorithm is Elliptical curve Diffie-Hellman that provides similar security with smaller key size. Elliptical curve is also quite popular and used with several blockchain systems and therefore for the exchange of session key, Elliptical curve Diffie-Hellman is used in the system. 


\section{The Elliptic Curve Diffie-Hellman Key Exchange:}

To implement the algorithm (see Figure 5), we need an elliptical curve $E$ with points $P=\left(X_{p}, Y_{p}\right)$ and a large prime number $p$. Once the session key $K_{\text {session }}$ is generated, the system can use session keys to encrypt or decrypt the data with the same key. The session key is $K_{\text {session }}$ that is equal to $a b=a(b P)=a b P$ and similarly for the other hand side $b A=b(a P)=a b P$. Therefore patient, doctors and system administrator or other staff can use this method to share the key over an insecure network. We use the session key $K_{\text {session }}$ for in encryption, digital signature and hashing throughout in our paper. Any user can create the session key by using this algorithm.

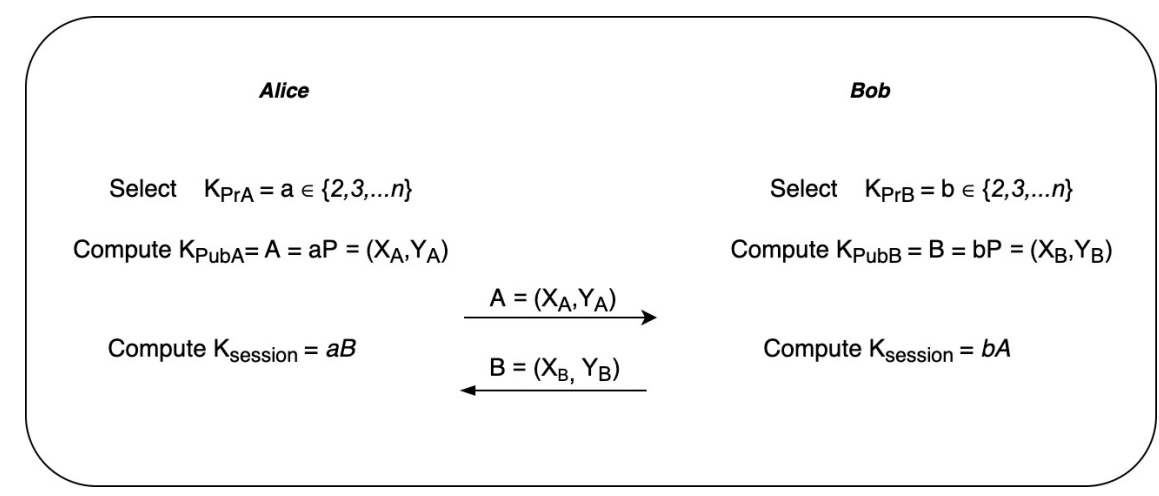

Fig. 5. Elliptic Curve Diffie-Hellman Key Exchange.

\section{Hash Function:}

A cryptographic hash function is a one-way function that is practically infeasible to invert. It absorbs data of arbitrary size and produce a fixed size output. Hash functions are mostly used for message authentication codes, digital signature and other authentications. A cryptographic hash function should have following properties:

- Pre-image resistance: Given a hash value $H$ of a message, it is infeasible to find the the original message. This is because hash functions are one way function and non-invertible.

- Second pre-image resistance: Given an input $m_{1}$, it is infeasible to calculate second input $m_{2}$ such that $\operatorname{hash}\left(m_{1}\right)=\operatorname{hash}\left(m_{2}\right)$.

- Collision resistance: It is infeasible to find two different messages $m_{1}$ and $m_{2}$, such that $\operatorname{hash}\left(m_{1}\right)=\operatorname{hash}\left(m_{2}\right)$.

\section{Digital Signature:}

For the purpose of authenticity and non-repudiation, a digital signature is used. In general, a private key of a given user is used to sign the document and users public key is used to verify. However, we are using hash based digital signature and use the session key $\left(K_{\text {session }}\right)$ to sign and verify the document. The process is explained with the help of Figure 6. 


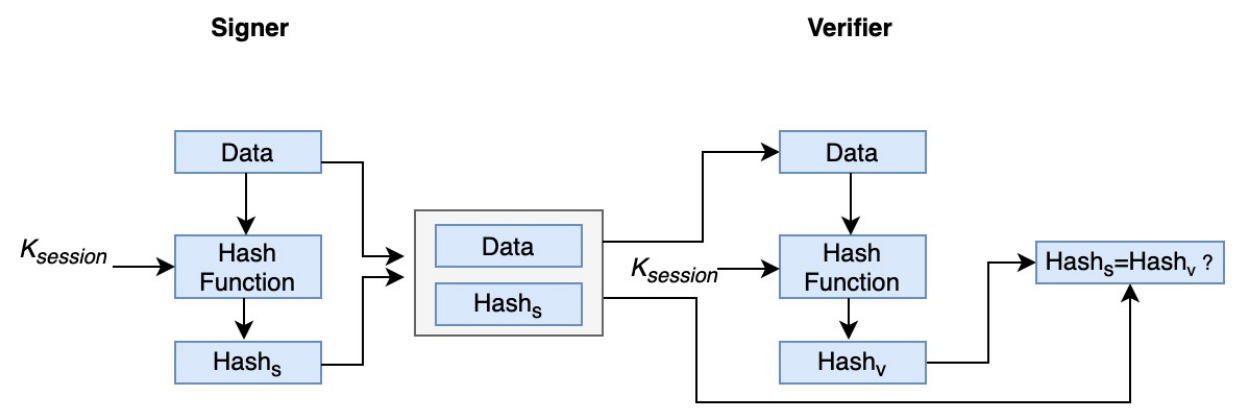

Fig. 6. Digital Signature

\subsection{Privacy information storage based on blockchain technology}

Although cloud computing technology uses cryptography and other related technologies, there are still weak links in the security of the entire cloud network. In particular, in terms of information privacy, at this stage, there is no sound legal protection system for medical privacy, and people do not have a strong sense of self-protection for personal information. The security of cloud computing is still troubling for any sort of sensitive data. In order to ensure the safe storage of MPI, the medical system privacy information storage system based on blockchain technology is designed. To detect the changes in the cloud data, blockchain is used. All events of system along with the hash of the cloud contents are stored on blockchain. Any changes on the cloud data can be easily traceable. The information encryption storage process is designed as follows:

(1) Medical institutions generate medical records and upload the metadata and summary of medical records to the blockchain. Medical record metadata includes: medical system privacy information ID and its uniform resource locators in the cloud database as well as other less important data relating to the EMR. The hash of the data is also stored on blockchain. A single bit of change in the cloud data will change the whole hash value and therefore any changes can be detectable.

(2) Medical records metadata are encrypted and transmitted safely to the patient after establishing the session key. Medical institutions adopt the method of encrypting MPI with AES encryption algorithm, and generate the session key $K_{\text {session }}$ using the Elliptic Curve Diffie-Hellman Key exchange, which effectively guarantees the security of MPI in the transmission process. When the patients registered in the hospital, hospital provides a unique ID and a file ID (File_HashID). The File_HashID is created by hashing the EMR of patients data and therefore always unique. Hospital also provide the folder_address of medical records and set the permission to access folder for particular user. The entire initial user information is represented by Equation (1). However, the File_HashID of the patient is created by the hashing of EMR that is expressed by Equation (2).

$$
\text { Patient }\left\{\text { ID, } \mathrm{K}_{\text {session, }} \text {, FolderAddress, Permission }\right\}
$$

$$
\operatorname{SHA}\left(\mathrm{K}_{\text {session }}, \mathrm{EMR}\right)=\text { File_HashID }
$$

The encryption and storage process is shown in Figure 7. In figure, the patient and hospital generate a session key $\left(K_{\text {session }}\right)$ and hospital use the key to encrypt data after completing the medical treatment. The hospital encrypts the patient's medical record file EMR by 


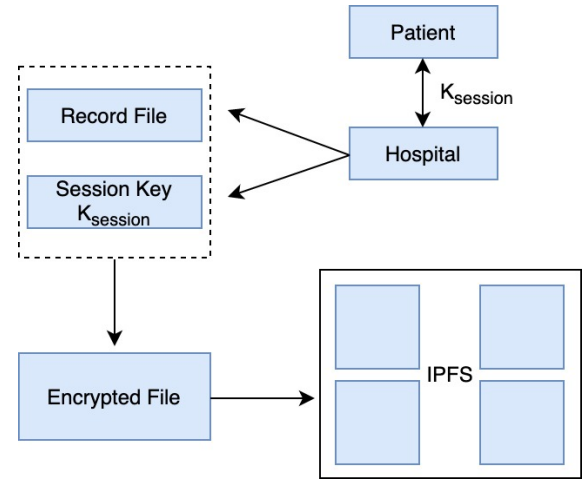

Fig. 7. File encryption and storage in IPFS

AES encryption. The encrypted record file is stored under the medical records folder address, FileAddress, for uploading to the IPFS service. The whole encryption process can be represented by Algorithm 1.



(3) The patient encrypts and uploads EMR to the medical cloud database. The patient and hospital generates a session key, encrypts the medical record with the key, and then uploads it to the cloud database for storage. All rights of privacy information in medical system belong to patients, and the rights of other users also need to be allocated by patients to control different users' access to privacy information.

This section describes the transmission process of an EMR hash and EMR encrypted file on the blockchain network and IPFS, respectively. The process is executed through an automatically executed smart contract. First, the EMR passed through the hash function SHA3 and encryption algorithm AES. SHA3 and AES produces the hash value and encrypted file of EMR. After receiving the hash value and encrypted file of EMR, the contract automatically transfers the file to the MPN 
(medical private network). The unique file ID (HashID1) of the file is obtained through the hashing and given by Equation (3) while encryption is given by Equation (4).

$$
\begin{aligned}
& \text { SHA } 3\left\{\mathrm { K } _ { \text { session } } \text { ,RecordFile } \left(\text { Pic }_{1}, \text { Pic }_{2}, \ldots, \text { Pic }_{n}, \text { Video }_{1}\right.\right. \text {, } \\
& \text { Video } \left.\left._{2}, \ldots, \text { Video }_{\mathrm{n}}, \text { Text }_{1}, \text { Text }_{2}, \ldots, \text { Text }_{\mathrm{n}}\right)\right\}=\text { HashID }_{1} \\
& \text { AES }\left\{\mathrm { K } _ { \text { session } } \text { RecordFile } \left(\text { Pic }_{1}, \text { Pic }_{2}, \ldots, \text { Pic }_{n}, \text { Video }_{1}\right.\right. \text {, } \\
& \text { Video } \left.\left._{2}, \ldots, \text { Video }_{\mathrm{n}}, \text { Text }_{1}, \text { Text }_{2}, \ldots, \text { Text }_{\mathrm{n}}\right)\right\}=\text { EMR }_{\text {Encrypted }}
\end{aligned}
$$

However, a complete EMR file often includes different types of medical records, such as: X-ray pictures, symptom videos, text of treatment results: The hash value of a folder contains multiple files. In this paper, the following symbols are used:

Pic $_{11}$, Pic $_{12}, \ldots$, Pic $_{1 n}$

Video $_{11}$, Video $_{12}, \ldots$, Video $_{1 n}$

Text $_{11}$, Text $_{12}, \ldots$, Text $_{1 n}$

If a patient is suffering from syndrome or multiple diseases, under an IPFS protocol, the same session key and patient ID can correspond to multiple folders but File_HashID will be changed because different file generate different hash value.

In this paper, $\operatorname{Hospital}_{A}\left(\right.$ File_HashID $_{1}$,File_HashID ${ }_{2} \ldots$ File_HashID $\left.{ }_{n}\right)$ is used, refers to the folder contained in the comprehensive disease during Hospital $_{A}$ visit.

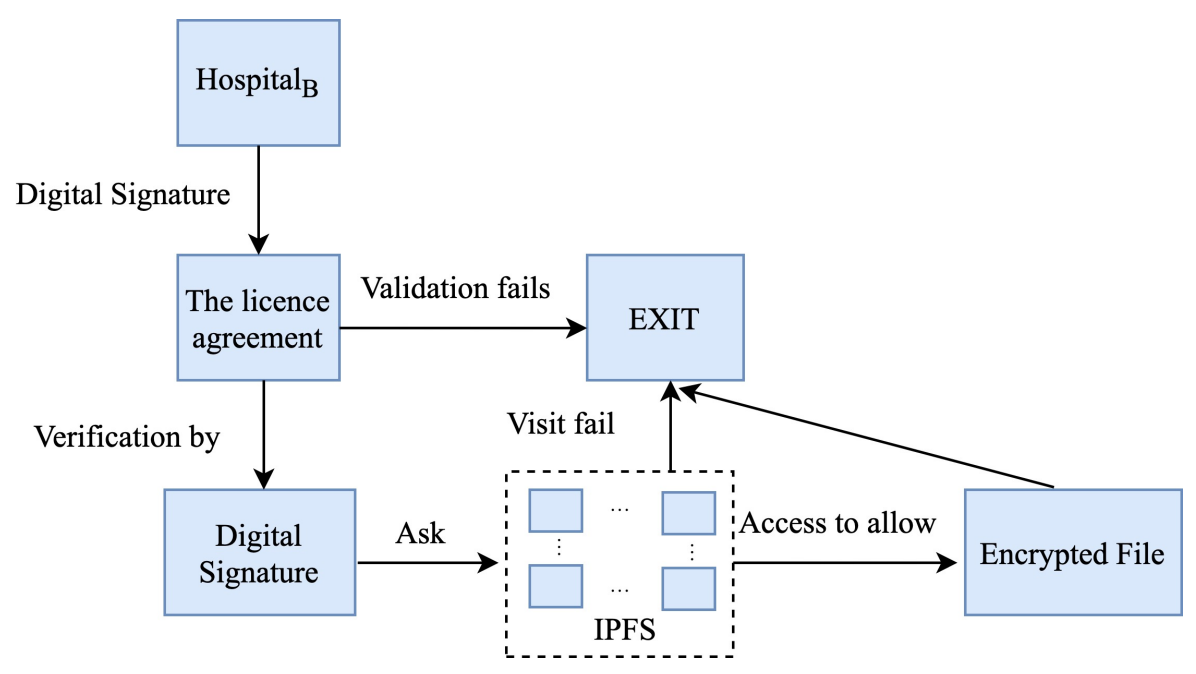

Fig. 8. Process of authorization contract execution

When $\mathrm{Hospital}_{B}$ needs to retrieve EMR, it first needs to verify the signature $\mathrm{Sig}_{B}$ of $\mathrm{Hospital}_{B}$ to determine it is a legal institution (see Figure 8). After passing the inspection, the Hospital $_{B}$ can access data of patient from Hospital $_{A}$. The contract execution process is as shown in Algorithm 2. 


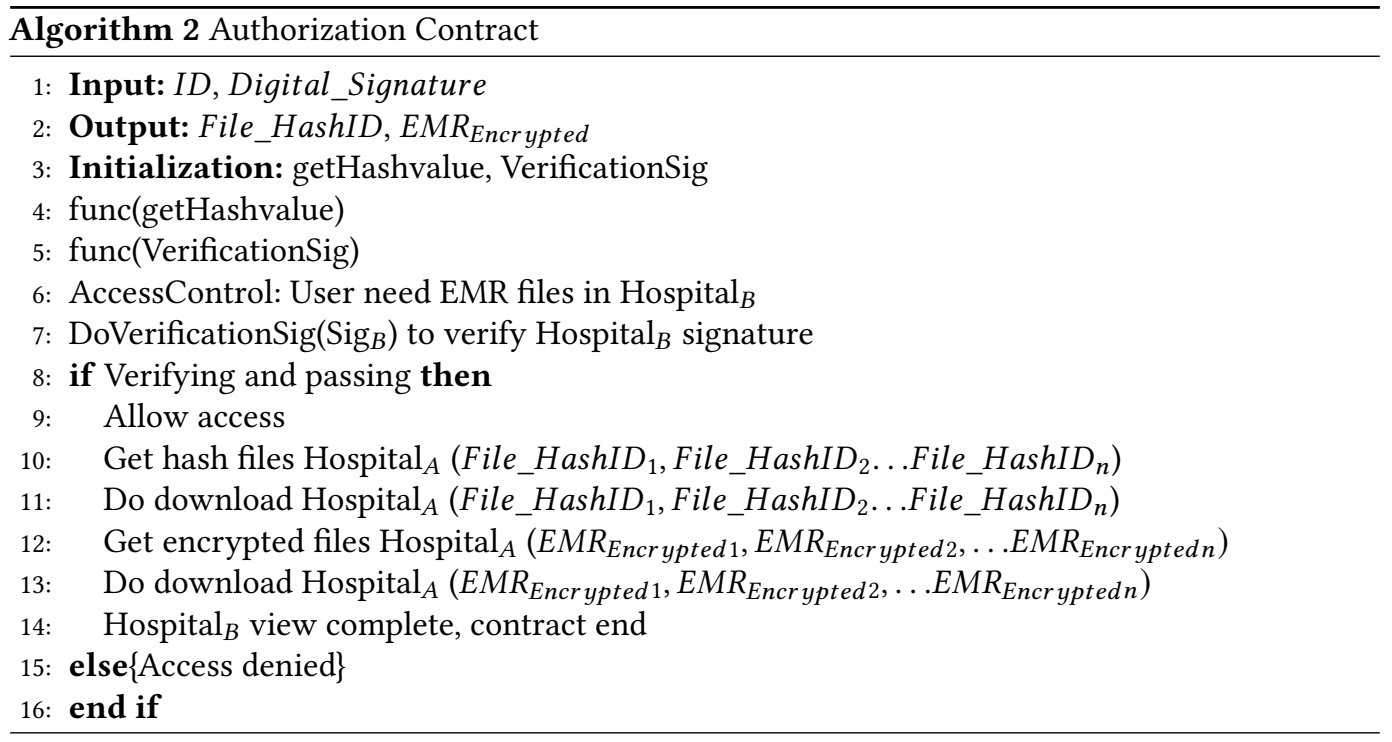

\subsection{Access control and security protection}

In order to implement personalized fine-grained privacy protection access control, the access control strategy is defined by the system administrator and the patient, which is divided into general strategy and personalized strategy. The system administrator is responsible for the binding of the role of the system user with the purpose of revocation and access. The patient's focus is on what purpose the personal privacy information is used for, so the main task is to specify the intention purpose of the information according to the personal privacy preference.

The general policy is: the access control policy defined by the system administrator according to the characteristics of his own organization and responsibility assignment. Its main task is to set the access purpose or the binding of the access purpose set related to the role and responsibility, and decide which permissions the role uses for which purposes. Through the definition of general policy, users who have passed the authentication can obtain the corresponding permissions of the role and access the allocated resources with reasonable purposes.

The general policy is a quadruple, which includes roles, operations, information resources and access purposes. It is formally defined as:

Common.Policy: $<\mathrm{r}$, op, ob, ap $>$, where $\mathrm{r} \in$ Roles, represents the role owned by the access requester, op $\in$ Ops represents operation, where ob $\in \mathrm{Obs}$, represents information resources, ap $\in \mathrm{AP}$, indicates the purpose of accessing information resources.

For example, the system administrator has defined a general strategy: emergency doctors should not view the patient's medical record information during emergency treatment, and then the strategy can be formally expressed as:

Common.Policy:<emergency doctor, not read, medical records, emergency treatment $>$.

The personalized strategy is: when patients are hospitalized for treatment and submit information to HIS system, the set of access rules defined according to their privacy preferences is designed to prevent unauthorized access to information and privacy disclosure.

Personalization strategy is a triplet, which includes information, operation and intention purpose. It is formally expressed as: 
Patient.Policy: $<$ Data, op, ip $>$, where Data represents the patient's privacy information, op $\in$ Ops represents the operation, and ip $\in$ IP represents the intention purpose specified by the patient for their privacy information.

For example, when Mary was in hospital, she defined a strategy. Her X-ray films can only be used for treatment purposes but not for other purposes (such as scientific research). Then the strategy can be formally expressed as:

Mary. Policy:<Mary. X-ray, Read, treat disease $>$.

\section{EXPERIMENTAL ANALYSIS}

For our experimentation validation, we used MATLAB $\vee 18.1$ and access the Amazon S3 (Simple Storage Service) by creating an account on Amazon Web Services (AWS). Amazon S3 can be used to store the file as on-line file storage. The data can be remotely accessed by anywhere around the world. The data stored on cloud can be used as datastore objects such as FileDatastore, ImageDatastore or SpreadsheetDatastore. To work with the Amazon S3 the first step is to setup the access:

- Sign up for AWS root account.

- Create an IAM (Identity and Access Management) by using the root account.

- Generate an access key and get the secret key and access key ID.

- Configure the machine with the help of secret key and access key ID.

Once the machine running MATLAB is connected to the cloud, we can use predefined functions to perform experiments on data. The method of this paper uses the symmetric key Algorithm AES for the encryption purpose and Elliptical curve Diffie-Hellman key exchange for the purpose of key generation on an in-secured transmission channel. Both methods are standard in cryptography and provide highest security. For the purpose of privacy of information, we used POPPAC model. The event of system and hash of the data is stored on blockchain and therefore any event performed by users or outsiders or any changes on the cloud data can be easily detected. In order to verify the effectiveness of the privacy information protection method based on blockchain, the following experiments are designed to verify. In a certain area, the clinical departments of an armor hospital take medical information as the experimental object, MATLAB software as the experimental environment, and use the methods of this paper, Malliaros' method (Method1 [20]), Alghazo's method (Method 2) [1] to compare the storage efficiency and throughput, and the security control performance of the three methods in the process of information protection [1,20]. Among them, storage efficiency and information throughput can initially verify the effectiveness of the method, security and security control performance can further verify the effectiveness of the method, and effectively prove the protection ability of the method to the privacy information of the medical system.

\subsection{Throughput comparison}

Throughput rate refers to the amount of data transmitted per unit of time. It is a measure of the data transmission rate of a computer or data communication system and can be used to comprehensively evaluate the capability of data storage or transmission methods to handle data requests. Therefore, in order to verify the data transmission performance of the proposed method, the application performance of the proposed method and the two comparison methods is analyzed from the perspective of information throughput. The higher the throughput is, the better the transmission performance of medical data is. The test data volume is defined between 50kbit and 550kbit. The statistical results of data transmission throughput of the three methods are shown in Figure 9. 


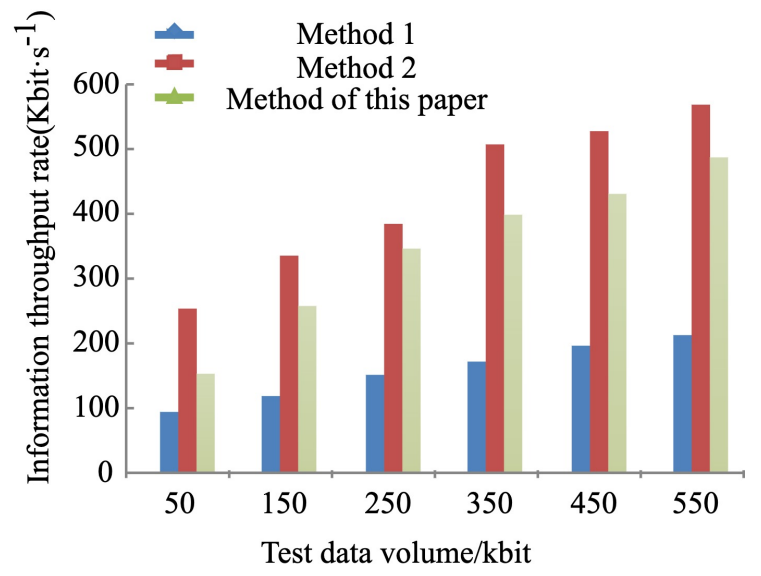

Fig. 9. Information throughput ratio comparison of different methods

In Figure 9, the information throughput of the three methods increases with the continuous increase in the amount of transmitted data. In contrast, the throughput rate of the proposed method is significantly higher than that of the two comparison methods, and the maximum information throughput rate reaches $550 \mathrm{kbit} / \mathrm{s}$, indicating that the proposed method has high data transmission efficiency and better transmission performance.

\subsection{Information security control performance comparison}

In order to further verify the security control performance and overall performance of the proposed method, three methods are compared from three aspects, namely, data cross-domain transmission reliability, broadband utilization and data cross-domain transmission network. The comparison results are shown in Table 2 .

Table 2. Comparison of safety control performance of different methods

\begin{tabular}{|l|l|l|l|}
\hline Methods & Method 1 & Method 2 & $\begin{array}{l}\text { Methods of } \\
\text { this paper }\end{array}$ \\
\hline $\begin{array}{l}\text { Data cross-domain transmission } \\
\text { reliability }\end{array}$ & Lower & Lower & Higher \\
\hline Bandwidth utilization & Lower & Lower & Higher \\
\hline $\begin{array}{l}\text { Cross-domain data transfer network } \\
\text { requirements }\end{array}$ & Higher & Higher & Lower \\
\hline
\end{tabular}

From Table 2, by comparing with the two literature comparison methods, the method proposed in this paper has higher reliability and bandwidth utilization of cross domain data transmission, and lower requirements for data transmission network. The results show that the method has high safety control performance and superior overall performance. This is because the method in this paper introduces POPPAC model and builds a private information storage platform by using blockchain. In addition, file synchronization and authorization contract are used to ensure the security of private information. 


\subsection{Safety performance comparison}

This paper uses symmetric encryption to encrypt data and asymmetric algorithm to transfer the session. As described in the previous section 2.3 our system provide the highest standard security with lightweight keys. When the user needs to access information of blockchain network or cloud, the authorization contract needs to be executed to check whether the user has access rights. In order to prove the security of private information under this method, the security performance of this method and two document comparison methods are analyzed from the aspects of information concentration, encryption time, decryption time, access control and ciphertext size. See Table 3 for comparison results.

Table 3. Comparison of security performance of different methods

\begin{tabular}{|l|l|l|l|}
\hline Methods & Method 1 & Method 2 & $\begin{array}{l}\text { Method of } \\
\text { this paper }\end{array}$ \\
\hline Information concentration & Yes & Yes & No \\
\hline Encryption time & $\mathrm{hT}_{i}$ & $\mathrm{~T}_{i}$ & $\mathrm{~T}_{i}$ \\
\hline Decryption time & $\mathrm{hT}_{j}$ & $\mathrm{~T}_{j}$ & $\mathrm{~T}_{j}$ \\
\hline Whether access control exists & $\mathrm{Yes}$ & $\mathrm{No}$ & Yes \\
\hline Ciphertext dimension Angle & $(3 \mathrm{~h}+1) \mathrm{N}$ & $\mathrm{h}^{2}+\mathrm{hN}+\mathrm{N}$ & $\mathrm{N}$ \\
\hline
\end{tabular}

In Table $3, \mathrm{~N}$ is the size of ciphertext data, $\mathrm{h}$ is the number of ciphertext attributes, $\mathrm{T}_{j}$ is the time spent decrypting 1 byte data, $T_{i}$ is the time spent encrypting 1 byte data. Based on security margin against various attacks, in our proposed model, we choose AES (Figure 10) cipher. There are some other lightweight encryption algorithms but they have not enough security margin against several attacks $[7,9,10]$. Also we are not using any IoT related devices in the system and therefore such encryptions are not necessary and our main goal is to find a secure and standard encryption algorithm.

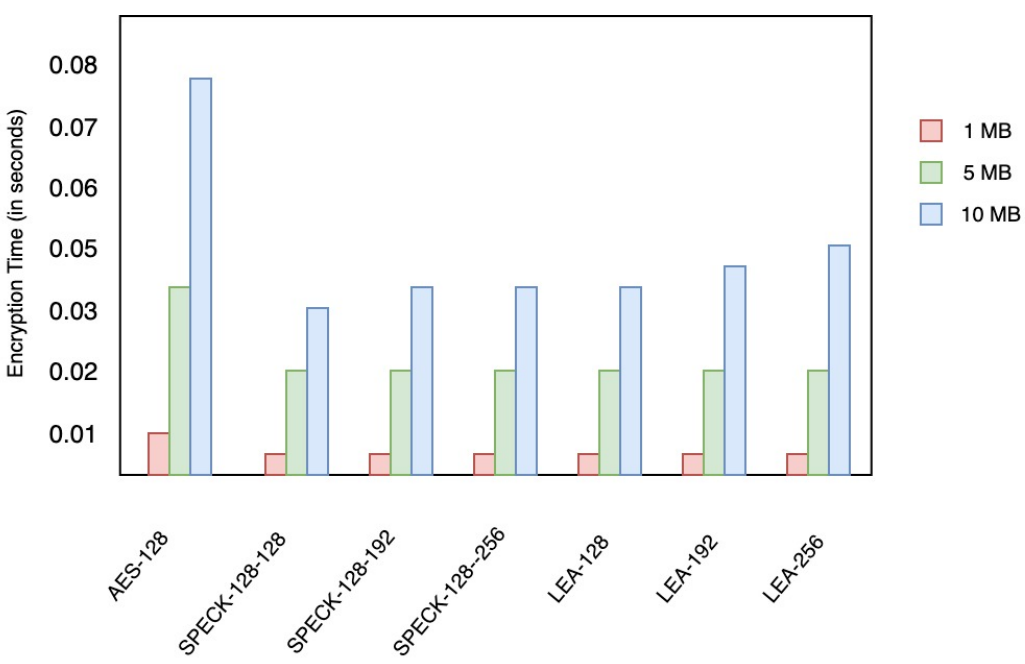

Fig. 10. Average AES encryption time (seconds). 
Since the ciphertext download in this paper does not depend on the number of data attributes, the time required in the encryption phase is $t_{i}$. Compared with the encryption time $H T_{i}$ of [14], the encryption time is shorter, and the same is true in the decryption phase. In terms of access control, [15] does not have an information access control strategy at present, but the access control strategy in this method can help users and other medical institutions quickly identify legitimate and illegal visitors. According to the size of ciphertext, the method proposed in this paper obtains EMR of multiple patients by downloading ciphertext after access control, saving bandwidth. In a word, compared with the traditional two methods, this method can significantly improve the information security.

\subsection{Storage Efficiency test}

The storage efficiency is reflected by the storage process response time. The shorter the response time is, the higher the storage efficiency is.

3.4.1 Storage efficiency of different types of information. Considering the practical application, two kinds of privacy information are selected, which are multimedia file and pure electronic document file. The method in this paper is used to store these two types of privacy information, and the storage efficiency statistics results are shown in Figure 11. In the figure, under the control of the method in this paper, the response time of the storage process of the pure text file information in the medical system differs little from that of the multimedia fusion file information. As the number of files increases, the pressure between network nodes increase, so does the response time of the stored process. In contrast, the response time required to store a multimedia file is greater than the time required to store a purely electronic document file. However, when using this method to store multimedia files, the highest response time is also below $1000 \mathrm{~ms}$, which indicates that this method has good control over the information storage process, high storage efficiency and no obvious blockage.

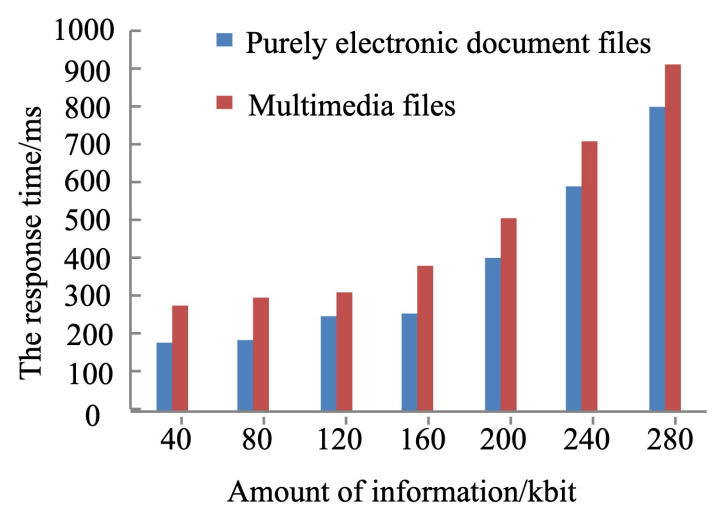

Fig. 11. Storage efficiency comparison

3.4.2 Storage efficiency comparison. In order to highlight the storage efficiency of this method, this method is compared with Methods 1-2. Three methods are used to store small files of $1000 \mathrm{~KB} 9000 \mathrm{~KB}$ and large files of $1000 \mathrm{~KB} 5000 \mathrm{~KB}$. The response time of different stored procedures is calculated, and the result is shown in Figure 12. As can be seen from Figure 12, the response time of the storage process of the method in this paper rises relatively slowly, indicating that the overall storage efficiency of the method in this paper is relatively stable and less disturbed by the change of file size. 

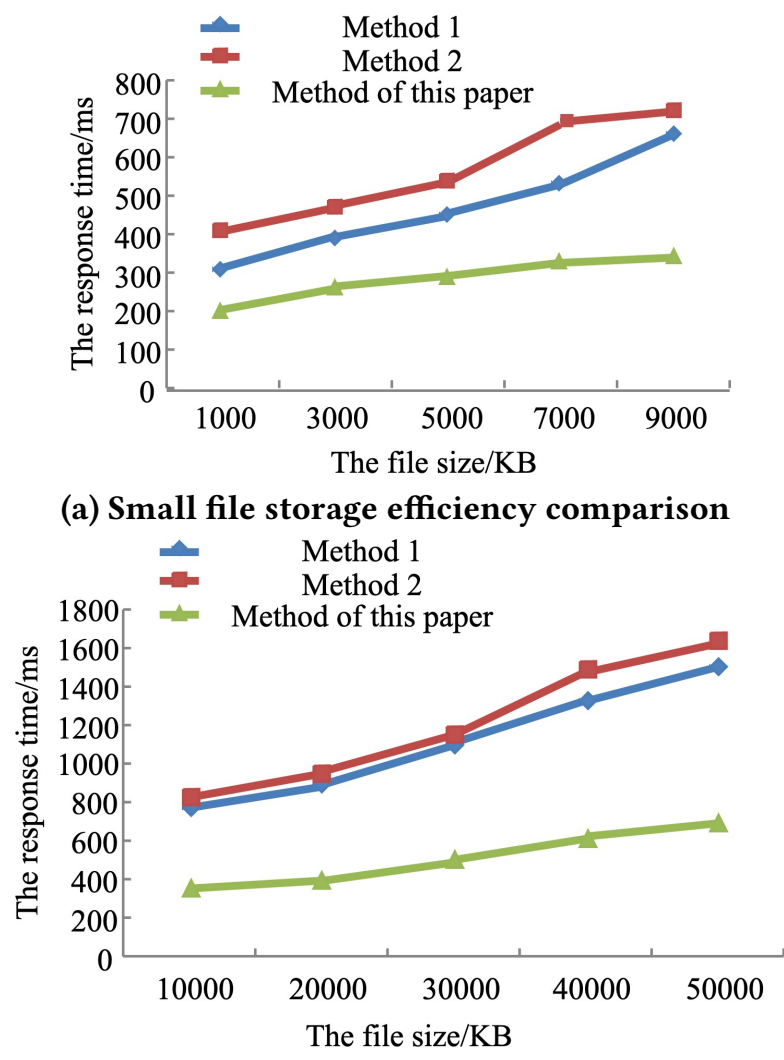

(b) Large file storage efficiency comparison

Fig. 12. Storage efficiency comparison of different methods

The response time of the two comparison methods in the storage of large files increases greatly, the storage efficiency is unstable, and the response time of the storage process is always more than the method in this paper. Therefore, the method proposed in this paper has a high efficiency in the storage of private information.

\section{DISCUSSION}

Blockchain and cloud database storage provide technical basis for the safe storage and sharing of medical system privacy information. The medical system privacy information platform designed in this paper can not only realize the distributed storage of medical system privacy information, but also ensure the right control of patients to medical privacy information, and realize the sharing application of third-party users to medical system privacy information. Patients, medical institutions and scientific research institutions can share medical information on this platform, such as disease diagnosis and treatment, genetics, diet, environment, lifestyle and other medical information, and on this basis, explore and develop new health care and disease prevention and treatment methods.

With the development of medical system privacy information, blockchain as a event storage technology will bring more opportunities and challenges to the development of medical. At present, the exploration and practice of policies, standards and other links is the primary task of the industry development. In view of this subversive change, taking a cautious and gradual approach is also a 
good way to apply blockchain in the medical field. The privacy protection and sharing system of electronic health archives based on blockchain can enable the data owner to achieve fine-grained access control for the data uploaded to the cloud, and the access control strategy based on blockchain records can better realize the sharing of health data. This paper proposes an effective control method for the security of privacy information in medical system. The advantages of this method in storage efficiency, throughput and security control performance are demonstrated through experimental comparison and analysis.

In this method, blockchain technology is mainly used in the privacy information protection scenario of medical system. In fact, this method can also be used in other common data protection and sharing scenarios, and the security of the method will not have any impact. Although this method proposes to solve some problems encountered in the process of privacy information protection of medical system based on blockchain technology, there are still many deficiencies that need to be focused on in the future.

(1) The initial version of blockchain, based on Proof of Work (PoW) suffers from too much energy consumption [23] in mining. Many consensus algorithms were proposed in response to that but all have their pros and cons.

(2) In the method, a model of access control strategy is proposed, but this strategy is a little rough. According to the needs of users, it is also an improvement to develop a more detailed access control model.

(3) Finally, through the experimental analysis, the storage efficiency, throughput and security control performance of the method are verified, but the storage cost is not evaluated, so in the future research, we need to build a practical block chain based privacy information protection and sharing system to achieve further improvement of the method.

Blockchain technology has outstanding advantages, but it also has defects. As a new technology, blockchain has some technical risks in its early application. When blockchain distributed structure stores and processes massive information, it puts forward higher requirements for hardware, and there is a bottleneck in its application in high-frequency trading industry. In the public chain, all data is open and transparent, and privacy is difficult to guarantee. In different fields of application, the attitude and position adopted by government departments also face severe challenges. The lag of legal system and regulatory measures relative to technology development may become the hotbed of blockchain technology applied in black industry in the regulatory gray area. In the wide application of blockchain in the future, the following problems need to be solved: technically optimizing transaction performance; reducing the deployment cost of application blockchain; ensuring the security of blockchain from the perspective of technology and management; establishing standards to increase the trust of blockchain; actively exploring the effective supervision of blockchain technology.

\section{CONCLUSION}

This paper proposes a method to protect the privacy information of medical system based on block chain technology, and tests the superiority of this method in the aspects of information storage and transmission efficiency and security control performance through simulation experiments. As a multi-party participation and multi-party supervision technology, blockchain technology can guarantee the traceability of stored records at any time, but there may be security problems such as key being exposed artificially in the transmission. Therefore, this paper applies the Elliptic Curve Diffie-Hellman Key Exchange to its internal storage information to provide effective protection for users' privacy information. The experimental results also prove that the proposed method is efficient in information storage and transmission and has high performance in information security 
control. In future research, the application effect of this technology in other fields should be further explored, and more encryption technologies such as state encryption and proxy encryption should be combined to improve the application of cryptography in blockchain technology and strengthen the protection of user privacy information.

\section{ACKNOWLEDGEMENTS}

The work of Ashutosh Dhar Dwivedi is funded by a grant from the Independent Research Fund Denmark for Technology and Production, grant no. $8022-00348 \mathrm{~A}$ and the work of Gautam Srivastava is funded by Natural Sciences and Engineering Council of Canada (NSERC) Discovery Grant held by Gautam Srivastava (RGPIN-2020-05363).

\section{REFERENCES}

[1] J. M. Alghazo. Intelligent security and privacy of electronic health records using biometric images. Current Medical Imaging, 15(4):386-394, 2019.

[2] J. Amini, A. H. Viki, A. Radan, and M. Moallem. A general control method for multilevel converters based on knapsack problem. IEEE Transactions on Power Electronics, 32(1):2-10, 2016.

[3] Z. BaynhamHerd. Enlist blockchain to boost conservation. Nature, 548(7669):523-523, 2017.

[4] M. Baza, N. Lasla, M. Mahmoud, G. Srivastava, and M. Abdallah. B-ride: Ride sharing with privacy-preservation, trust and fair payment atop public blockchain. IEEE Transactions on Network Science and Engineering, 2019.

[5] J. C. Chrisler, A. Barney, and B. Palatino. Ageism can be hazardous to women's health: ageism, sexism, and stereotypes of older women in the healthcare system. Fournal of Social Issues, 72(1):86-104, 2016.

[6] J. Daemen and V. Rijmen. Rijndael/aes. In H. C. A. van Tilborg, editor, Encyclopedia of Cryptography and Security. Springer, 2005.

[7] D. A. Dhar, P. Morawiecki, and S. Wójtowicz. Finding differential paths in arx ciphers through nested monte-carlo search. International fournal of Electronics and Telecommunications, vol. 64(No 2), 2018.

[8] A. D. Dwivedi, L. Malina, P. Dzurenda, and G. Srivastava. Optimized blockchain model for internet of things based healthcare applications. In 2019 42nd International Conference on Telecommunications and Signal Processing (TSP), pages 135-139. IEEE, 2019.

[9] A. D. Dwivedi, P. Morawiecki, and G. Srivastava. Differential cryptanalysis of round-reduced SPECK suitable for internet of things devices. IEEE Access, 7:16476-16486, 2019.

[10] A. D. Dwivedi and G. Srivastava. Differential cryptanalysis of round-reduced LEA. IEEE Access, 6:79105-79113, 2018.

[11] A. D. Dwivedi, G. Srivastava, S. Dhar, and R. Singh. A decentralized privacy-preserving healthcare blockchain for iot. Sensors, 19(2):326, 2019.

[12] P. Fairley. Blockchain worldfeeding the blockchain beast if bitcoin ever does go mainstream, the electricity needed to sustain it will be enormous. IEEE Spectrum, 54(10):36-59, 2017

[13] J. J. Fenton, J. A. Robbins, A. L. D. Amarnath, and P. Franks. Osteoporosis overtreatment in a regional health care system. JAMA internal medicine, 176(3):391-393, 2016.

[14] H. Huang, X. Chen, and J. Wang. Blockchain-based multiple groups data sharing with anonymity and traceability. Science China Information Sciences, 63(3):130101, 2020.

[15] E. Krah, J. de Kruijf, and L. Ragno. Integrating traditional healers into the health care system: Challenges and opportunities in rural northern ghana. Journal of community health, 43(1):157-163, 2018.

[16] S. Liu, W. Fu, L. He, J. Zhou, and M. Ma. Distribution of primary additional errors in fractal encoding method. Multimedia Tools and Applications, 76(4):5787-5802, 2017.

[17] S. Liu, C. Guo, F. AlTurjman, K. Muhammad, and V. H. C. de Albuquerque. Reliability of response region: A novel mechanism in visual tracking by edge computing for iiot environments. Mechanical Systems and Signal Processing, 138:106537, 2020.

[18] S. Liu, Z. Pan, and X. Cheng. A novel fast fractal image compression method based on distance clustering in high dimensional sphere surface. Fractals, 25(04):1740004, 2017

[19] Z. Ma, W. Huang, and H. Gao. Secure drm scheme based on blockchain with high credibility. Chinese fournal of Electronics, 27(5):1025-1036, 2018.

[20] S. Malliaros, C. Xenakis, G. Moldovan, J. Mantas, A. Magdalinou, and L. Montandon. The integrated holistic security and privacy framework deployed in crowdhealth project. Acta Informatica Medica, 27(5):333, 2019.

[21] M. E. Peck and S. K. Moore. The blossoming of the blockchain. IEEE Spectrum, 54(10):24-25, 2017.

[22] Y. Qi and Y. Huang. Dira: Enabling decentralized data integrity and reputation audit via blockchain. Sci. China Technological Sci., 62:698-701, 2019. 
[23] R. Singh, A. Dwivedi, G. Srivastava, A. Wiszniewska-Matyszkiel, and X. Cheng. A game theoretic analysis of resource mining in blockchain. Cluster Computing: The fournal of Networks, Software Tools and Applications, 2020.

[24] N. R. Sperber, R. A. Bruening, A. Choate, E. Mahanna, V. Wang, B. J. Powell, T. Damush, G. L. Jackson, C. H. Van Houtven, K. D. Allen, et al. Implementing a mandated program across a regional health care system: A rapid qualitative assessment to evaluate early implementation strategies. Quality Management in Healthcare, 28(3):147-154, 2019.

[25] G. Srivastava, A. D. Dwivedi, and R. Singh. Automated remote patient monitoring: Data sharing and privacy using blockchain. CoRR, abs/1811.03417, 2018.

[26] G. Srivastava, R. M. Parizi, A. Dehghantanha, and K.-K. R. Choo. Data sharing and privacy for patient iot devices using blockchain. In International Conference on Smart City and Informatization, pages 334-348. Springer, 2019.

[27] P. Treleaven, R. G. Brown, and D. Yang. Blockchain technology in finance. Computer, 50(9):14-17, 2017.

[28] S. Underwood. Blockchain beyond bitcoin, 2016.

[29] J. J. Wyse, A. J. Gordon, S. K. Dobscha, B. J. Morasco, E. Tiffany, K. Drexler, F. Sandbrink, and T. I. Lovejoy. Medications for opioid use disorder in the department of veterans affairs (va) health care system: Historical perspective, lessons learned, and next steps. Substance abuse, 39(2):139-144, 2018.

[30] A. Yazdinejad, G. Srivastava, R. M. Parizi, A. Dehghantanha, K.-K. R. Choo, and M. Aledhari. Decentralized authentication of distributed patients in hospital networks using blockchain. IEEE fournal of Biomedical and Health Informatics, 2020.

[31] X.-M. Zhang, Q.-L. Han, and X. Ge. A novel finitesum inequalitybased method for robust $h_{\text {infty }}$ control of uncertain discretetime takagi-sugeno fuzzy systems with intervallike timevarying delays. IEEE transactions on cybernetics, 48(9):2569-2582, 2017.

[32] B. Zhao, Y. Xiao, Y. Huang, and X. Cui. A private user data protection mechanism in trustzone architecture based on identity authentication. Tsinghua Science and Technology, 22(2):218-225, 2017.

[33] D. Zhaoyang, L. Fengji, and G. Liang. Blockchain: a secure, decentralized, trusted cyber infrastructure solution for future energy systems. Journal of Modern Power Systems and Clean Energy, 6(5):958-967, 2018. 